\title{
Computational Chemistry and Non-noble Metal-catalyzed Cross-coupling Reaction Mechanisms
}

\author{
Jeremy N. Harvey* and Andrea Darù
}

\begin{abstract}
This short overview discusses the contribution that computational methods can make to the study of reaction mechanisms in non-noble-metal-catalyzed cross-coupling reactions. The differences between such reactions and those catalyzed by more traditional noble metals are emphasized, together with the challenge this poses to computation.
\end{abstract}

Keywords: Computational chemistry $\cdot$ Cross-coupling $\cdot$ Non-noble metal catalysis

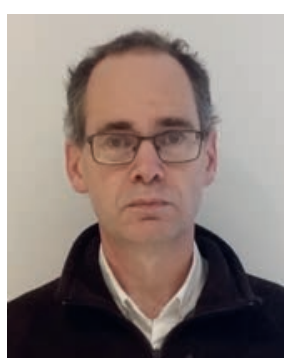

Jeremy $N$. Harvey was born in the UK and educated in Belgium, gaining his $\mathrm{PhD}$ in organic chemistry with Heinz Viehe in 1995. After postdoctoral periods in Germany (Helmut Schwarz, TU Berlin) and Israel (RB Gerber, Jerusalem), he became a lecturer at the University of Bristol, where he remained until 2014, when he moved to his current position in Leuven. His research interests are in computational chemistry and chemical reaction mechanisms.

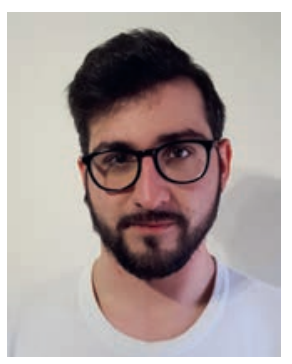

Andrea Darù studied chemistry at the University of Ferrara, then carried out postgraduate experimental and computational research in the group of Prof. Merino at the University of Zaragoza in Spain. After obtaining a postgraduate degree in 2015 , his developing passion for computational chemistry and reaction mechanisms led him to move to KU Leuven to join the group of Prof. Harvey. In Leuven Andrea was involved in a Marie Skłodowska-Curie ITN project aimed at studying non-noble metal catalysis that is the subject of his $\mathrm{PhD}$ thesis (gained in 2020).

\section{Introduction}

Non-noble metals (earth-abundant metals such as iron or nickel and others from the first transition metal row) are attracting increasing interest for their various applications in homogeneous catalysis, including cross-coupling reactions. Many of the reactions catalyzed by such metals remain poorly understood in mechanistic terms. Here, we will give a short overview of the state of quantum chemical computational studies of these crosscoupling catalytic reactions involving non-noble metal catalysts.

Since the early days of computational organometallic chemistry, there has been considerable interest in elementary steps involved in catalysis based on metals such as palladium or platinum. In fact, the very first $a b$ initio geometry optimization of a transition state for a reaction of an organometallic species was carried out $^{[1]}$ for a simple model reaction relevant to platinum catalysis:

${ }^{\star}$ Correspondence: Prof. J. N. Harvey, E-mail: Jeremy.harvey@kuleuven.be Department of Chemistry, KU Leuven, Celestijnenlaan 200F, B-3001 Leuven, Belgium

$$
\mathrm{Pt}\left(\mathrm{PH}_{3}\right)_{2}+\mathrm{H}_{2} \rightarrow \mathrm{Pt}(\mathrm{H})_{2}\left(\mathrm{PH}_{3}\right)_{2}
$$

Since then, there have been a large number of studies of such computational studies, ${ }^{[2]}$ focusing either on individual elementary steps such as oxidative addition, or on whole catalytic cycles. This is thereby almost a mature field, where the contribution that computational methods can make is well-known. Much of the work in the area is based on the use of density functional theory, as this represents a good compromise between accuracy and computational expense. However, more and more often, correlated $a b$ initio methods are used, which offer the promise of getting much more accurate results. We do not intend to suggest that there are no remaining challenges in the field of computational homogeneous catalysis for noble metal catalysts. It remains very difficult to make firm predictions concerning e.g. improved catalysts, or to predict a reaction mechanism in the absence of extensive experimental data concerning intermediates and substituent effects. Nevertheless, with our growing knowledge of the issues arising in computational studies of this type, more and more pertinent results are being obtained. ${ }^{[3]}$

Computational studies for cross-coupling involving non-noble metals are much less numerous. This is partly because the experimental work in the field has so far been less abundant, but also because there are considerable challenges involved in the study of such reactions. Foremost among these challenges is that nonnoble metals tend to follow rather different mechanistic pathways. Organometallic chemists are used to drawing mechanisms involving steps such as ligand addition or dissociation, oxidative addition, reductive elimination, and migratory insertion. Indeed, some cross-coupling reaction mechanisms with non-noble metal catalysts do seem to operate using such steps. ${ }^{[4]}$ However, many other cross-coupling reactions appear to operate with rather different mechanisms, in particular because the 'traditional' organometallic reaction steps typically involve changes in oxidation state by increments of two, whereas non-noble metals often have stable oxidation states different by increments of just one.

Another point of contrast between 'noble' metal catalysts and non-noble metals is that catalysis with the former quite often involves relatively simple reaction conditions. The word 'relatively' is emphasized because cross-coupling chemistry is notorious for sensitivity to the nature of the ligand on the metal, additives such as bases used to react with departing protons or other Lewis acidic species, and the type of metal catalyst precursor used. We do not wish to minimize this complexity! Still, with non-noble metals, 
it is quite common to encounter aspects such as the use of solid metal reductants, or large excesses of nucleophile, or sensitivity to the presence of Lewis acids, which cannot readily be accounted for in simple mechanistic terms.

Against this background, we will discuss some notable successes in the field and some of the challenges.

\section{Selected Computational Studies}

One of the earliest thorough computational studies of crosscoupling with a non-noble metal was carried out in the group of Norrby in 2009. ${ }^{[5]}$ This remarkable paper combines some new experimental results with a computational attempt to map out possible mechanisms. In their study, Norrby et al. first used experiments to assess the likely oxidation state of the key active species, as well as computational studies to investigate relative energies for key TSs, for iron-catalyzed Kumada-type coupling of $p$-trifluoromethyl phenyl chloride and $n$-octylmagnesium bromide, in ether or amide solvent. This is a variant of the reaction that was developed by Tamura and Kochi in the 1970s. ${ }^{[6]}$ Among the computational steps that they considered were the following reductive elimination processes (where DME is dimethylether, as a model for the ether solvents used):

$$
\mathrm{Fe}(\mathrm{DME})_{2}\left(\mathrm{C}_{2} \mathrm{H}_{5}\right)(\mathrm{Ph}) \rightarrow \mathrm{PhC}_{2} \mathrm{H}_{5}+\mathrm{Fe}(\mathrm{DME})_{2}
$$

$$
\mathrm{Fe}(\mathrm{Cl})(\mathrm{DME})_{2}\left(\mathrm{C}_{2} \mathrm{H}_{5}\right)(\mathrm{Ph}) \rightarrow \mathrm{PhC}_{2} \mathrm{H}_{5}+\mathrm{Fe}(\mathrm{Cl})(\mathrm{DME})_{2}
$$

The first of these reactions involves a starting species with a + II oxidation state for the iron centre, leading to an $\mathrm{Fe}(\mathrm{o})$ oxidation state for the product, and the second reaction involves oxidation states $+\mathrm{III}$ and $+\mathrm{I}$ respectively. Likewise reductive elimination steps involving initial oxidation states for iron of - II and -I were also considered. Based on the calculated energetics, it was concluded that the two steps shown are those that are most likely to be involved in catalysis, though other evidence pointed towards the +III/+I step as more likely. Also, reaction (3) was suggested to be more likely, as reaction (2) had a very high activation barrier.

This study was a landmark in the study of reaction mechanisms with non-noble metals using computation, because it explicitly addressed the huge mechanistic uncertainty for such reactions, and set out to try to provide some input into possible mechanisms by carrying out sensitivity tests for some key reaction steps, varying the oxidation state and exploring the dependence of reaction barriers on the oxidation state. In the authors' view, with large uncertainties about the mechanism, this kind of broad overview of possibilities based on simple computational models provides more mechanistic information than detailed studies of assumed catalytic cycles.

Still, it should be recognized that this study did not - and most likely could not - pin down the correct mechanism, as that mechanism is rather complex. Building on earlier work by Kochi and other groups, the group of Mike Neidig showed that a polynuclear $\mathrm{Fe}_{8} \mathrm{R}_{n}(n=12)$ cluster is apparently important in the Kumadacoupling. ${ }^{[7]}$ Such polynuclear species had not been considered in the computational study.

While reactivity studies for organometallic catalysis naturally often focus on the study of TSs and reaction paths, for non-noble catalysts, there is also very significant interest in the electronic structure of intermediates and TSs, due to the frequency with which open-shell electronic structures occur. Probing potential intermediates using spectroscopic methods is very helpful in such cases, and computation can really assist in understanding the spectral properties. In our group, we carried out a number of such studies in collaboration with the experimental group of Robin Bedford at the University of Bristol in the UK, putting considerable effort into calculating the structure and EPR parameters of putative $\mathrm{Fe}(\mathrm{I})$ and related species involved in cross-coupling. ${ }^{[8]}$ Even simple aspects like the ground-state spin-pairing of each intermediate is not always obvious to assign based on experiment alone, and calculations can help to suggest the correct spin state (for an example from another group, see ref. [9]).

As mentioned above, cross-coupling with non-noble metal catalysts does not need to follow the same mechanisms as for more common cross-coupling reactions. One nice example of a study ${ }^{[10]}$ providing strong evidence for a different, bimetallic radical-like mechanism used computation in part to support a Kumada coupling mechanism in which the key carbon-carbon bond forming steps were (here $\mathrm{L}$ is a ligand):

$$
\mathrm{LFe}^{\mathrm{II}} \mathrm{Ph}+\mathrm{R}^{\cdot} \rightarrow \mathrm{LFe}^{\mathrm{III}} \mathrm{PhR}
$$

$$
\mathrm{LFe}^{\mathrm{III}} \mathrm{PhR} \rightarrow \mathrm{R}-\mathrm{Ph}+\mathrm{L}^{\mathrm{I}} \mathrm{Fe}
$$

This mechanism involves $\mathrm{Fe}(\mathrm{I})$ and $\mathrm{Fe}(\mathrm{III})$ species, and has as key $\mathrm{C}-\mathrm{C}$ bond-forming step reaction (5), a reductive elimination from $\mathrm{Fe}(\mathrm{III})$, but formation of the key $\mathrm{Fe}(\mathrm{III})$ intermediate involves multiple steps and free radicals - rather unlike the traditional oxidative addition and transmetallation steps expected in more conventional Kumada couplings. One such key step is reaction (4). It is not yet clear how general such mechanisms are, with for example the dependence on ligands, or on the $\mathrm{sp}^{2}$ or $\mathrm{sp}^{3}$ hybridization of the organometallic species and the halide still requiring more investigation. Yet other mechanisms may also be operative.

Cross-coupling with nickel catalysts is also often suggested, based on computational studies, to involve formation of radicals during activation of the $\mathrm{C}-\mathrm{X}$ bond..$^{[11]}$ Here too, it is unclear to what extent the radical nature of these reactions is a common feature. At least in some early studies, oxidative addition steps similar to those found with palladium have been characterized also for $\mathrm{Ni}$, at least in the case of the Heck reaction. ${ }^{[12]}$ This has also been confirmed in more recent studies. [13]

In our recent work, carried out during the $\mathrm{PhD}$ studies of one of us (AD), we have tried to approach a 'whole-mechanism' computational study of challenging non-noble metal-catalyzed crosscoupling reactions. By 'whole-mechanism', we mean that we tried to go beyond providing insight into individual intermediates, or individual elementary steps, and instead to provide an overall picture of the whole cross-coupling mechanism. Our work of this type has so far led to two publications, whose broader themes we will briefly summarize here.

The first study ${ }^{[14]}$ concerned the mechanism of reaction (6) between an alkyl iodide and an alkyne, developed recently in a collaborator's group. ${ }^{[15]}$
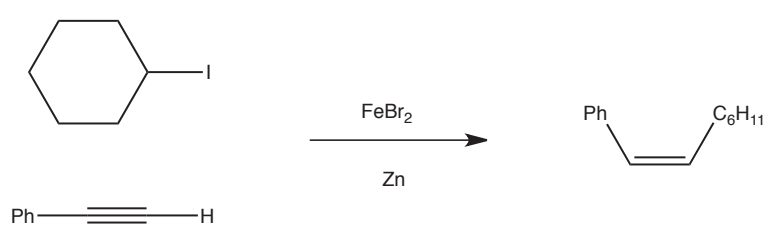

$\mathrm{Zn}$

The challenge in modelling this reaction was considerable, as there was no ligand involved, and relatively little mechanistic data concerning possible (or excluded) mechanistic intermediates. In our work, we focused on, among other things, finding a way to address the thermodynamics of reduction by solid zinc for the dissolved iron-containing species, on determining the spin- and solvation-state of the various iron-containing species, and on exploring the variety of possible reaction steps that could lead to carbon-carbon bond formation. We also used kinetic modelling to assess which collection of reaction steps most likely accounted for observed reactivity. Our conclusion was that the mechanism 
was best described by a reductive cleavage step (7) of the carboniodine bond, driven by zinc but presumably modified by the presence of iron salts:

$$
\mathrm{C}_{6} \mathrm{H}_{11}-\mathrm{I}+1 / 2 \mathrm{Zn} \rightarrow \mathrm{C}_{6} \mathrm{H}_{11}+1 / 2 \mathrm{ZnI}_{2}
$$

This would be followed by addition of the cyclohexyl radical to the alkyne. While our study was not able to resolve all the mechanistic issues for this complicated reaction, we did provide a road-map for techniques to study complex cross-coupling reactions of this type.

Our second study ${ }^{[16]}$ addressed the coupling of a nitrogencontaining species, typically a diazo-compound $\mathrm{ArN}_{2} \mathrm{Ar}$, with an ester, to provide an amide. This is not strictly a cross-coupling reaction, though it shares many features with this class of reactions. It is catalyzed by nickel salts with phenanthroline ligands and involves metallic zinc as a reductant to create the nitrogen nucleophile. Using some of the same techniques as in the other study, we were able to quantify the thermodynamics of reductive steps involving metallic zinc, and to provide evidence that the key step in the reaction involved a bimetallic nickel-zinc amidate.

\section{Conclusions}

To conclude this short overview, we wish to emphasize that while computational chemistry is going to be a key technique for studying cross-coupling reaction mechanisms involving non-noble metal catalysts, it is striking that the 'chemical' space of possible mechanistic alternatives and of viable intermediates seems to be much larger than for more standard palladium-catalyzed reactions. ${ }^{[17]}$ Multiple spin-states, coordination states, oxidation states, seem to play an important role. Some of the reactions we have modelled feature as important steps reduction by a metallic co-reactant. Also, radicals play an important role in many of the mechanisms. Hence although individual computational analyses as cited here provide insight into individual reactions, it is very difficult to obtain a global overview of the whole mechanism. As in many cases, the strongest basis for getting such global insight will be firm kinetic and mechanistic input from experiment for some typical processes, coupled with systematic computational studies using accurate quantum chemical methods in order to obtain reliable results. Furthermore, it is important to realize that non-noble metals are quite often different in terms of catalytic mechanisms from more standard metals.

\section{Acknowledgements}

We gratefully acknowledge generous financial support from the European Union Marie Sklodowska-Curie Initial Training NoNoMeCat (675020-MSCA-ITN-2015-ETN).
[1] K. Kitaura, S. Obara, K. Morokuma, J. Am. Chem. Soc. 1981, 103, 2891, doi: $10.1021 / \mathrm{ja00400a079}$

[2] For one of many reviews on computational studies of Pd-catalyzed crosscoupling, see L. Xue, Z. Lin, Chem. Soc. Rev. 2010, 39, 1692, doi: 10.1039/ b814973a.

[3] J. N. Harvey, F. Himo, F. Maseras, L. Perrin, ACS Catal. 2019, 9, 6803, doi: 10.1021/acscatal.9b01537.

[4] For an example of Suzuki-Miyaura cross-coupling with a cobalt catalyst, see J. M. Neely, M. J. Bezdek, P. J. Chirik, ACS Cent. Sci. 2016, 2, 935, doi: 10.1021/acscentsci.6b00283.

[5] J. Kleimark, A. Hedsström, P.-F. Larsson, C. Johansson, P.-O. Norrby, ChemCatChem 2009, 1, 152, doi: 10.1002/cctc.200900061.

[6] M. Tamura, J. Kochi, J. Am. Chem. Soc. 1971, 93, 1487, doi: 10.1021/ ja00735a 030 .

[7] See J. D. Sears, S. B. Muñoz III, S. L. Daifuku, A. A. Shaps, S. H. Carpenter, W. W. Brennessel, M. L. Neidig, Angew. Chem. Int. Ed. 2019, 58, 2769, doi: 10.1002/anie.201813578, and refs therein.

[8] For two selected examples, see: R. B. Bedford, P. B. Brenner, E. Carter, J. Clifton, P. M. Cogswell, N. J. Gower, M. F. Haddow, J. N. Harvey, J. A. Kehl, D. M. Murphy, E. C. Neeve, M. L. Neidig, J. Nunn, B. E. R. Snyder, J. Taylor, Organometallics 2014, 33, 5767, doi: 10.1021/om500518r; C. J. Adams, R. B. Bedford, E. Carter, N. J. Gower, M. F. Haddow, J. N. Harvey, M. Huwe, M. Á. Cartes, S. M. Mansell, C. Mendoza, D. M. Murphy, E. C. Neeve, J. Nunn, J. Am. Chem. Soc. 2012, 134, 10333, doi: 10.1021/ ja303250t.

[9] F. E. Zhurkin, M. D. Wodrich, X. Hu, Organometallics 2017, 36, 499, doi: 10.1021/acs.organomet.6b00841.

[10] G. Bauer, M. D. Wodrich, R. Scopelliti, X. Hu, Organometallics 2015, 34, 289, doi: $10.1021 /$ om501122p

[11] M. S. Cheung, F. K. Sheong, T. B. Marder, Z. Lin, Chem. Eur. J. 2015, 21, 7480, doi: $10.1002 /$ chem. 201500110.

[12] B.-L. Lin, L. Liu, Y. Fu, S.-W. Luo, Q. Chen, Q.-X. Guo, Organometallics 2004, 23, 2114, doi: 10.1021/om034067h.

[13] V. H. Menezes da Silva, A. A. C. Braga, T. R. Cundari, Organometallics 2016, 35, 3170, doi: 10.1021/acs.organomet.6b00532.

[14] A. Darù, X. Hu, J. N. Harvey, ACS Omega 2020, 5, 1586, doi: 10.1021/ acsomega.9b03578.

[15] C. W. Cheung, F. E. Zhurkin, X. Hu, J. Am. Chem. Soc. 2015, 137, 4932, doi: 10.1021 /jacs.5b01784.

[16] M. L. Ploeger, A. Darù, J. N. Harvey, X. Hu, ACS Catal. 2020, 10, 2845, doi: 10.1021/acscatal.9b05049.

[17] This point was also made in a review on Nickel cross-coupling, S. Z. Tasker, E. A. Standley, T. F. Jamison, Nature 2014, 509, 299, doi: 10.1038/nature 13274 .

\section{License and Terms}

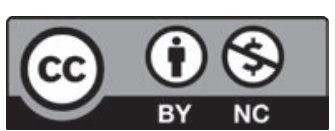

This is an Open Access article under the terms of the Creative Commons Attribution License CC BY_NC 4.0. The material may not be used for commercial purposes.

The license is subject to the CHIMIA terms and conditions: (http:// chimia.ch/component/sppagebuilder/?view=page \&id=12).

The definitive version of this article is the electronic one that can be found at doi:10.2533/chimia.2020.467 